Research Article

\title{
Hydrodynamic Analysis of the Flow inside the Submerged Entry Nozzle
}

\author{
Jesus Gonzalez-Trejo $\left(\mathbb{D},{ }^{1}\right.$ Cesar Augusto Real-Ramirez $\left(\mathbb{D},{ }^{1}\right.$ Ignacio Carvajal-Mariscal $\left(\mathbb{D},{ }^{2}\right.$ \\ Florencio Sanchez-Silva $\mathbb{D D}^{2}{ }^{2}$ Francisco Cervantes-De-La-Torre $\mathbb{D}^{\mathbb{D}},{ }^{1}$ Raul Miranda-Tello $\mathbb{D}^{\mathbb{D}}{ }^{1}$ \\ and Ruslan Gabbasov ${ }^{1}{ }^{1}$
}

${ }^{1}$ Universidad Autonoma Metropolitana, Mexico City, Mexico

${ }^{2}$ Instituto Politecnico Nacional, Mexico City, Mexico

Correspondence should be addressed to Cesar Augusto Real-Ramirez; carr@correo.azc.uam.mx

Received 14 August 2020; Revised 30 September 2020; Accepted 7 October 2020; Published 21 October 2020

Academic Editor: Ahmad Zeeshan

Copyright (C) 2020 Jesus Gonzalez-Trejo et al. This is an open access article distributed under the Creative Commons Attribution License, which permits unrestricted use, distribution, and reproduction in any medium, provided the original work is properly cited.

\begin{abstract}
The quality of steel produced by continuous casting depends mainly on the characteristics of the liquid steel flow pattern within the mold. This pattern depends on the flow dynamics of the nozzle that is immersed in liquid steel. This work characterizes the fluid dynamics within two separate submerged entry nozzle models with a square cross section bore. The Froude similarity criterion and water as working fluid have been used. The models consist of a square-shaped tube with one inlet and two lateral squared exits at the bottom. To enhance the flow visualization, the models do not have exit ports. Moreover, one of the models has a "pool," a volume at the bottom, and the other prescinds of it. The geometrical parameters and operational conditions of physical experiments were reproduced in the numerical simulations. The turbulence model used in this work is large eddy simulation (LES) with dynamic $k$-equation filtering. It was found that transient numerical simulations reproduce the dynamic nature of the internal flow pattern seen in physical experiments. The results show that the flow pattern within the pool nozzle is defined by only one large vortex; on the other hand, in the nozzle, without the pool, the flow pattern achieves a complex behavior characterized by two small vortexes. This study will allow to build nozzles that produce a symmetric, regular fluid flow pattern inside the mold, which leads to improvements on the process such as low energy consumption and finally in cost reductions.
\end{abstract}

\section{Introduction}

Molten steel continuous casting is an industrial process whose origins can be traced almost 200 years ago. Since the US Patent No. 1908 obtained by George Escol Sellers on December 17, 1840, titled "Machinery for making pipes continuously from lead," great experience has been gained in the operation of this process [1]. The continuous casting process can be seen in detail in the work [2]. Many theoretical and experimental investigations of the continuous casting process mainly aimed at obtaining high-quality homogeneous slabs were carried out. The aspect in which all research works have coincided is that the flow pattern of the jets of liquid steel emerging from the submerged entry nozzle (SEN) determines to a large extent the quality and purity of the steel slabs. The SEN is designed to regulate the continuous flow of liquid steel that is supplied to the mold [3]. Several recent investigations have concurred with the fact that properties that characterize the internal and external geometry of the nozzle influence the flow pattern that the SEN produces inside the continuous casting mold. There are, however, differences in the influence that each of these geometric properties has on the flow pattern of liquid steel inside the nozzle and within the mold. For example, Calderon-Ramos et al. (2019) [4] studied the effect of both the transverse shape of the exit ports and the angle of inclination of the ports. They showed that the square-shaped ports produce symmetric and slow jets. Similarly, Zhang et al. (2019) [5] studied the influence of two fundamental aspects of the SEN exit ports on the liquid steel flow pattern within 
the mold: the horizontal angle of inclination and the angle at which the ports' outlets diverge. The latter authors found an optimal combination of the port angle and immersion depth that reduces mold level fluctuations $[6,7]$. Common to most of these works is the investigation at the same time of several factors that modify the flow pattern inside the mold $[8,9]$. This approach, however, does not allow to separate the effects that each of them has on the flow inside the mold. On the other hand, previous works paid little attention to the exploration of the interior flow structure inside the SEN $[10,11]$.

The exit flow structure is developed in the bottom zone of the nozzle. However, factors such as the impurities dragged by liquid steel erode the internal walls which modify the internal volume of the SEN and thus the flow structure. The nozzles with a "pool," a volume at the bottom, suffer from a constant accumulation of the slag and impurities that fill the volume and change the fluid dynamics.

In order to numerically reproduce the flow pattern of the SEN, RANS and LES turbulence models were used previously to reproduce the internal flow pattern of the SEN $[4,12,13]$. In these works, it is possible to observe that RANS models could reproduce the average flow pattern; however, they do not obtain the transient dynamic flow behavior $[2,5-7,9,14-19]$. On the other hand, the LES turbulence model reaches accurate results, and it is possible to compare their results with the physical experimental data [20-31].

Additionally, the flow behavior presented in this work has similarities to the fluid flow behavior in other science and engineering fields, for example, the problem of mixing and vortex formation in $\mathrm{T}$-junctions found in pipes, arteries, venous systems, and microfluidic systems [31, 32].

The present work explores, using physical and numerical modeling, the effect of the well depth inside the SEN on the behavior of the jets that emerge from the nozzle ports. The box-type nozzle studied in this work has an inner bore with a square cross section that remains constant throughout all its length $[16,33]$. In order to reproduce only the inner walls of the SEN, we model them as a thin shell that we termed nozzle internal prototype (NIP). The NIP employed in this work has two exit ports of square geometric shape. Since we analyze only the bifurcation of the flow of steel in nozzle interior, the effects of inert gas injection, port angle, and thickness of the port were not considered. We carry out both physical and numerical simulations of NIP that would correspond to a real $1: 1 / 4$ scaled bifurcated SEN, based on the Froude hydrodynamic similitude criterion.

\section{Physical Simulations}

2.1. Nozzle Internal Prototypes Geometry. Figure 1 shows the NIP of two bifurcated SENs: one of them with a well (Figure 1(a)) and the other without a bottom well (Figure 1(b)), which will be named Case A and Case B, respectively. In addition, Figure 1 (c) shows the photography of the experimental NIP corresponding to Case B. In this figure, there can be seen all the relevant geometrical aspects for this study: inner bore geometry, exit ports geometry, bore inner length, and depth of the bottom well. It is worth noting that both NIP studied have a flat bottom.

With regard to geometry, these prototypes have a square inner bore with $0.02 \mathrm{~m}$ per side and two square-shaped ports with a side length of $0.014 \mathrm{~m}$. The areas of the two output ports sum roughly the transversal area of the bore nozzle. The depth of the nozzle bottom well for Case A is $0.007 \mathrm{~m}$, which is half the height of the exit ports.

The volumetric flow used was $1.5 \mathrm{~m}^{3} / \mathrm{h}$. This flow is close enough to that occupied in previous works $[3,18]$. Based on the Froude similitude criterion and considering that the working fluid is water, the NIPs used in the present study are $1: 1 / 4$ scaled models. The physical properties of the simulation fluid (water at $293.15 \mathrm{~K}$ ) are listed in Table 1.

2.2. Physical Simulation Description. Physical simulations were conducted under two distinct operating conditions. In all cases, the NIP discharged into a visualization cell, which is a rectangular prism with a square-shaped base of $0.015 \mathrm{~m}$ per side and a height of $0.5 \mathrm{~m}$. The characteristics of the experimental setup are similar to those used in [26].

In the first operating condition, the NIP discharges both exit jets freely into the atmosphere, and following Gupta and Lahiri (1992) [11], this operating condition will be named as a "free-fall jet condition." This configuration allows better visualization of several significant phenomena, such as the shape, size, and direction of the outlet jets, as well as visualizes the behavior of the fluid flow inside the bifurcated nozzle between the exit ports $[2,12,26]$.

In the free-fall jet condition, a high-speed camera was used to record the experiment at a rate of $1000 \mathrm{fps}$. The camera axis remains perpendicular to the axis formed by the centers of the exit ports. The camera was slightly tilted which permits better visualization of the NIP interior.

In the second operating condition, the NIP is submerged into the liquid. Following Gupta and Lahiri (1992) [11], this operating condition will be named as a submerged nozzle condition. The immersion depth measured between the free surface and the upper edge of the nozzle exit ports is $0.07 \mathrm{~m}$. In this operating condition, a digital camera was used to take multiple pictures. The camera axis was aligned with the axis formed by the centers of the nozzle exit ports, such that the camera lens plane is parallel to the plane of the exit ports.

In the submerged nozzle condition, an approach called "light painting" was implemented to visualize the vortex structures generated inside the NIP. Light painting is a photography technique that records the movement of a light source by taking a long exposure photo. In our case, the light sources are hollow glass particles of the same type as those used in PIV studies, illuminated by a continuous wave $3 \mathrm{~W}$ power laser that generates a $0.001 \mathrm{~m}$ thick plane.

\subsection{Physical Simulations: Case A}

2.3.1. Free-Fall Jet Condition. Figure 2 depicts a single vortex that forms on the bottom of the NIP captured at regular time intervals. The vortex rotation axis is almost parallel to the axis of the exit ports. This vortex emerges from the 


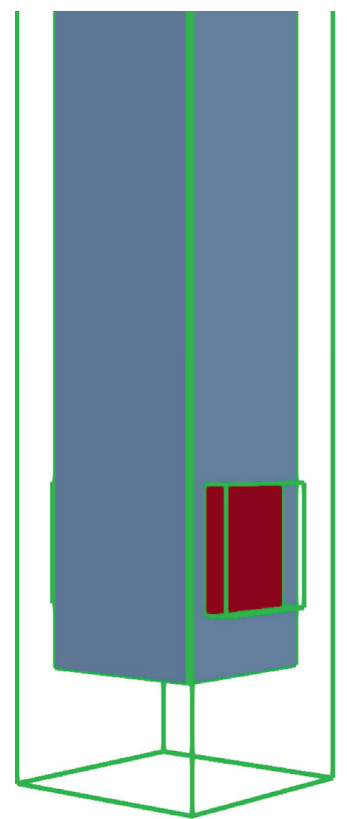

(a)

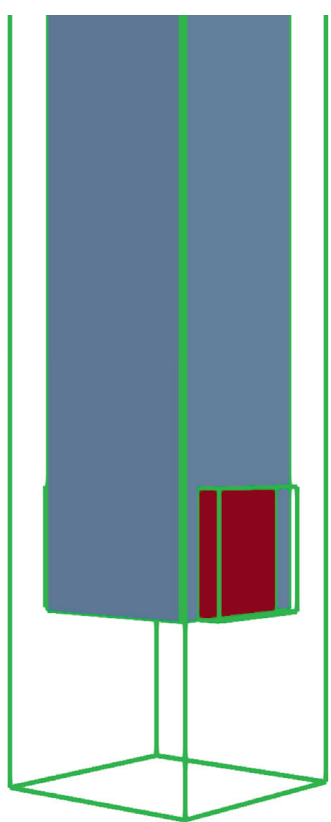

(b)

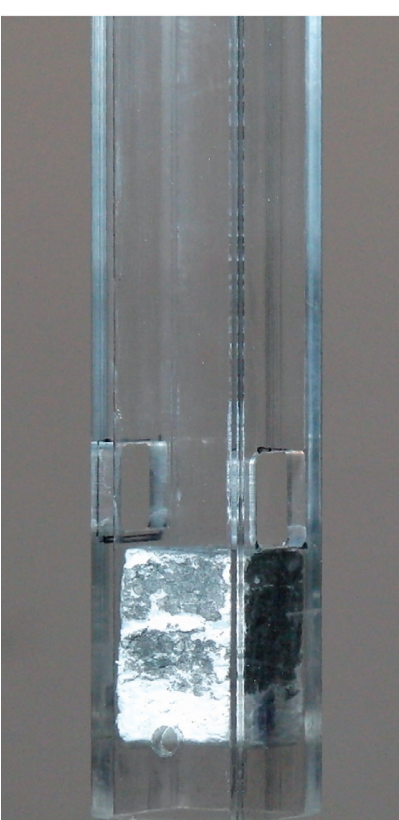

(c)

Figure 1: Representation of studied NIPs. (a) Numerical model of Case A. (b) Numerical model of Case B. (c) Photograph of Case B physical model.

TABLE 1: Physical properties of the simulation fluid.

\begin{tabular}{lc}
\hline Property & Value \\
\hline Dynamic viscosity, $\mu(\mathrm{Kg} / \mathrm{m} \cdot \mathrm{s})$ & $1 \cdot 10^{-3}$ \\
Density, $\rho\left(\mathrm{Kg} / \mathrm{m}^{3}\right)$ & $1 \cdot 10^{3}$ \\
Kinematic viscosity, $v\left(\mathrm{~m}^{2} / \mathrm{s}\right)$ & $1 \cdot 10^{-6}$ \\
Superficial tension, $\sigma(\mathrm{N} / \mathrm{m})$ & $7.5 \cdot 10^{-2}$ \\
\hline
\end{tabular}

development of low-pressure zones and acts as a siphon, suctioning surrounding air through small regions at the exit ports. Therefore, the vortex at the bottom of the nozzle inner bore becomes visible and outlines some of the reverse flow zones developing inside of bifurcated SEN. Previously, several authors have discussed the influence that the reverse flow phenomenon has on the formation of an asymmetric flow pattern over the course of time, inside the mold of the slab continuous casting machine $[14,21]$.

Figure 2 illustrates the features and phenomena of the hydrodynamic evolution of the vortex inside the NIP throughout $0.05 \mathrm{~s}$. Also, the hydrodynamic evolution of the heights of the upper and lower edges of the vortex inside the nozzle is plotted, which illustrates complex dynamic behavior with high-frequency components. The heights of the two edges were measured on the center plane of the nozzle. The plot includes the measurements for all frames in the 0.05 s time lapse.

Several six-second recordings were carried out; all of them had vortices with the same rotation direction. Nevertheless, high-speed videos have shown that at several times, the vortex breaks and loses continuity between the exit ports. The vortex rupture at that moment is caused by the increasing pressure values inside the reverse flow zone, as well as the ceasing of siphoning effect. In addition, these recordings register that the semicylindrical shape of the vortex frequently bends and sinks into the nozzle pool.

The images suggest that nozzle exit jets have not the same size. Indeed, the jet on the left side, most of the time, has a higher divergence angle than the jet on the right side. However, there are instants, where jet sizes become similar or the jet on the right side is broader than the jet on the left. These variations in the sizes and intensities of the jets will surely induce an asymmetrical behavior of the liquid steel flow pattern inside the continuous casting mold.

During the physical simulations, it was recorded a couple of air bubbles in the liquid stream which allowed a rough estimation of the flow velocity (Figure 3). Two bubbles traveled near the NIP central line, but their behavior near the vortex is distinct. When the vortex absorbs the right-hand side bubble (encircled in red), this one leaves the nozzle by the top of the exit port. Conversely, the left-hand side bubble (encircled in blue) surrounds the vortex and leaves the nozzle by the bottom of the exit port. If we assume that each bubble moves on a plane that is parallel to the observer, then the downstream velocity can be measured from the video 

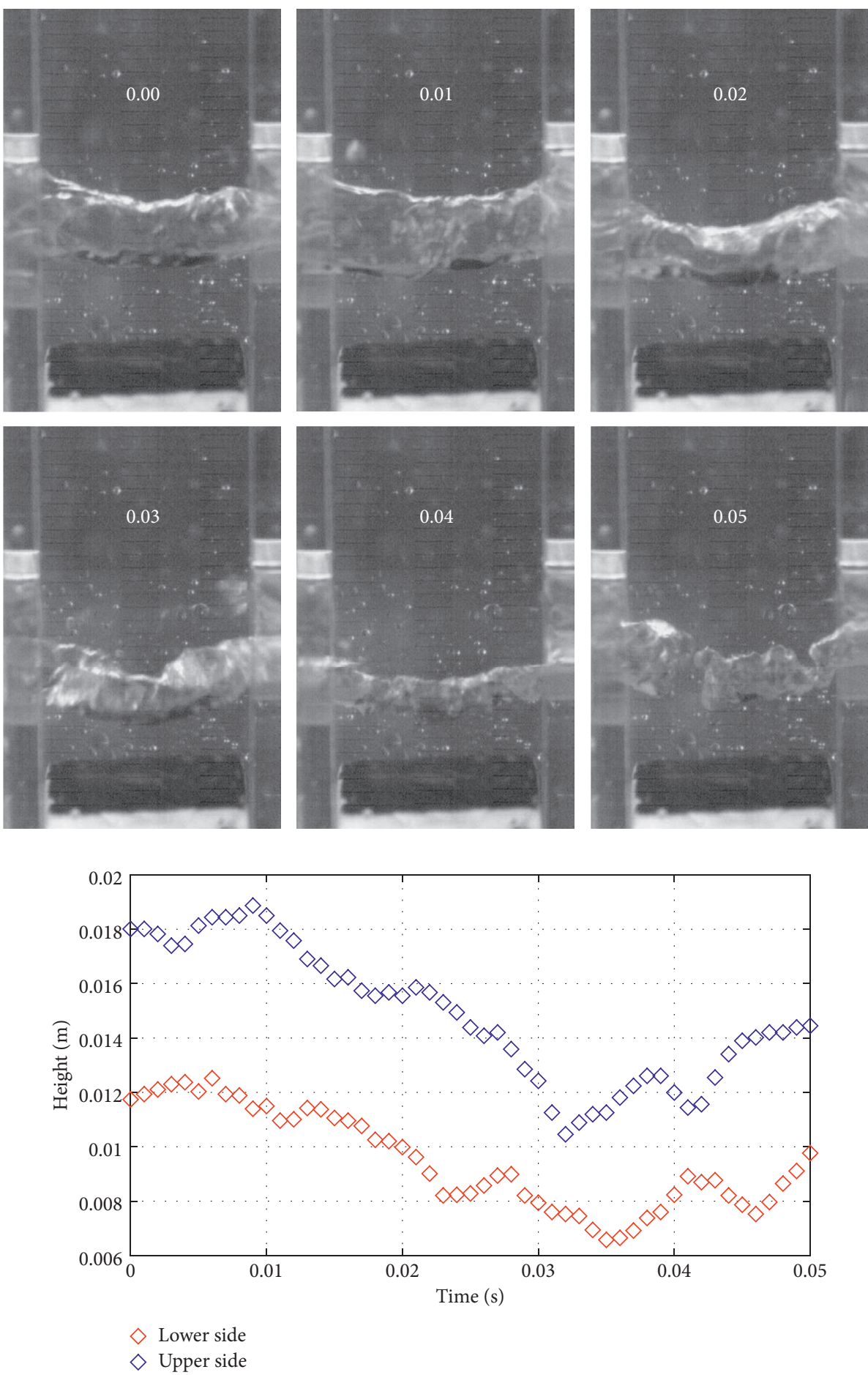

FIgURE 2: Dynamic evolution of the vortex inside the NIP, Case A, for a time interval of $0.05 \mathrm{~s}$ (upper panel). Also, in the bottom panel, the oscillation of the vortex position as its upper and lower side is shown.

recording as shown on the plot of the bottom of Figure 3. In both cases, the velocity grows as the bubbles approach the bottom of the NIP.
2.3.2. Submerged Nozzle Condition. In this operating condition, the NIP is submerged such that the jets are discharging into the liquid. In this case, also a single 

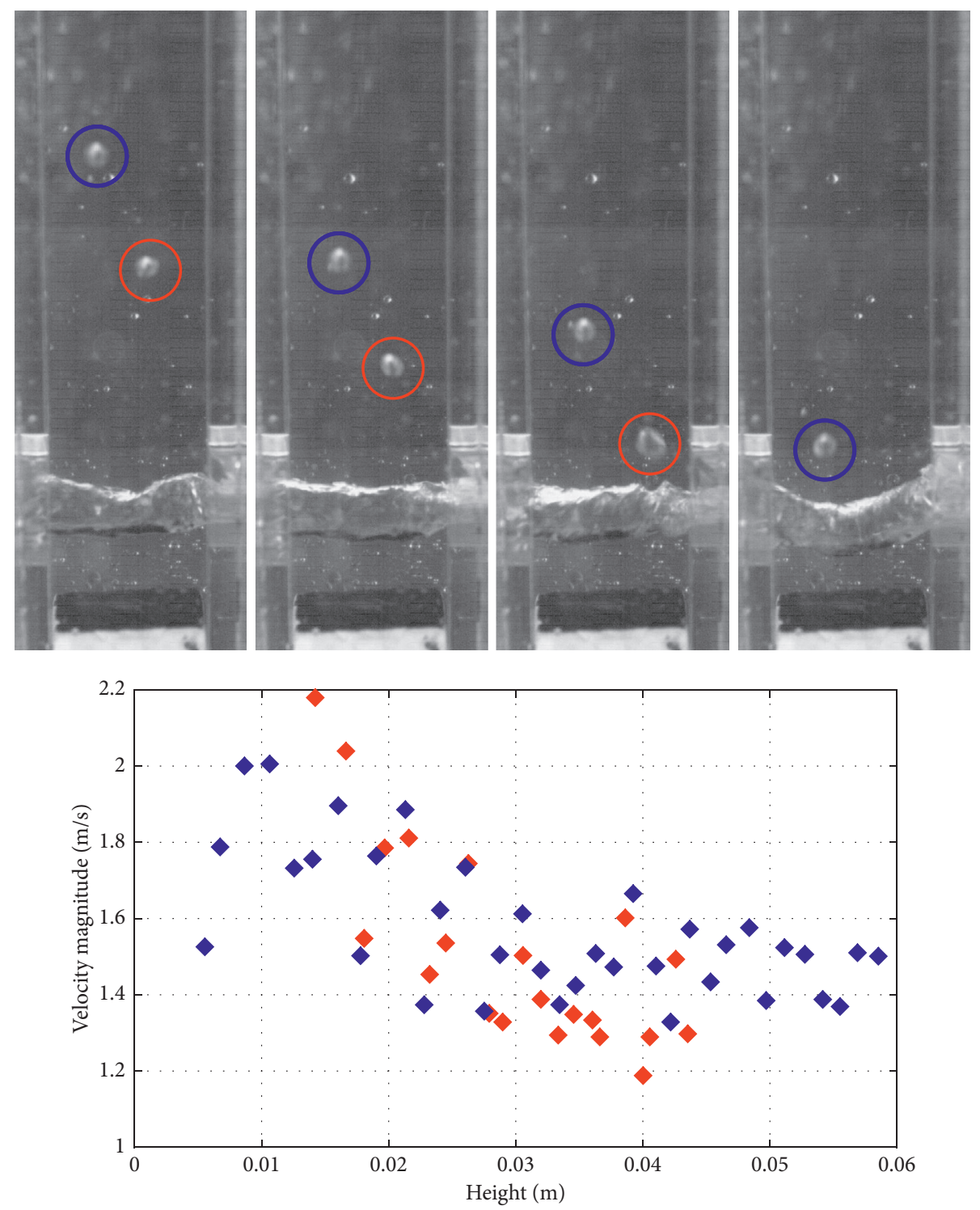

Figure 3: Dynamic evolution of two bubbles in Case A (top) and the velocities of the bubbles at different heights from the bottom of the nozzle (bottom).

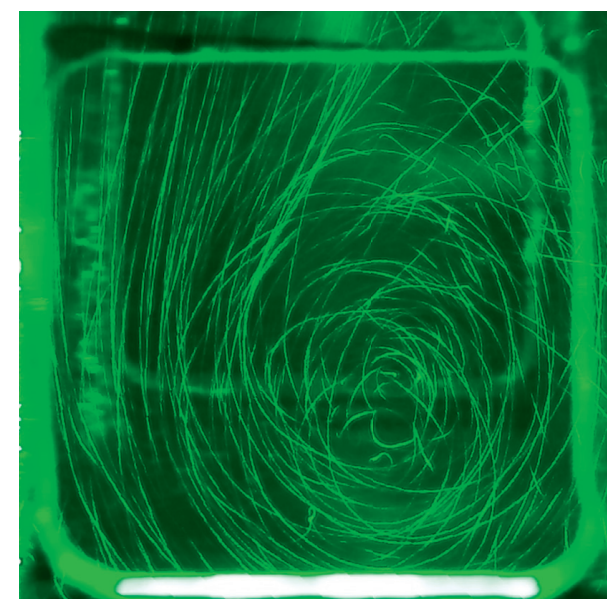

FIGURE 4: Vortical fluid flow pattern viewed from one of the exit ports for Case A submerged NIP. pronounced vortex is formed. Figure 4 shows a close-up of the vortex with its rotation axis parallel to the exit ports' axis. The photograph confirms the fact that the vortex axis rotation does not remain static while the camera diaphragm is open. Notice also that the vortex occupies a considerable area of the exit ports.

\subsection{Physical Simulations: Case B}

2.4.1. Free-Fall Jet Condition. The flow behavior of the NIP without a bottom well is very different from the previous case. Two counterrotating vortices are now formed inside the prototype. The high-speed video recording shows that there are several significant differences between the vortices observed in the NIP with and without well. The first difference is that the two vortices of the NIP without bottom well rarely connect both exit ports entirely. Another 


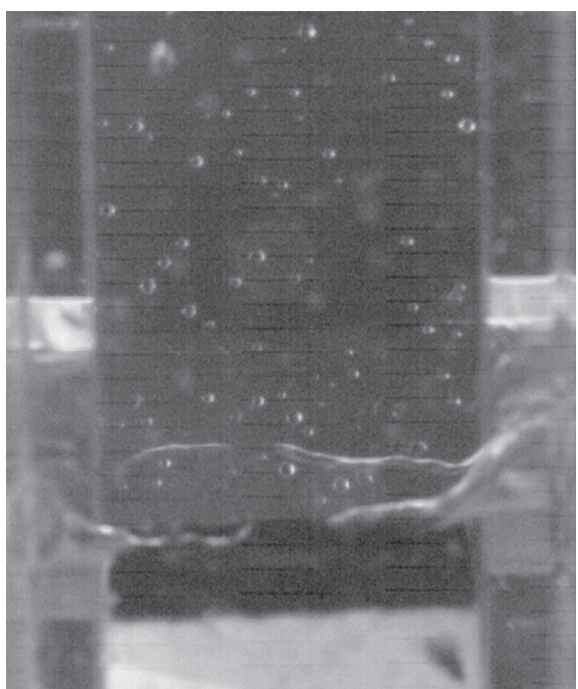

(a)

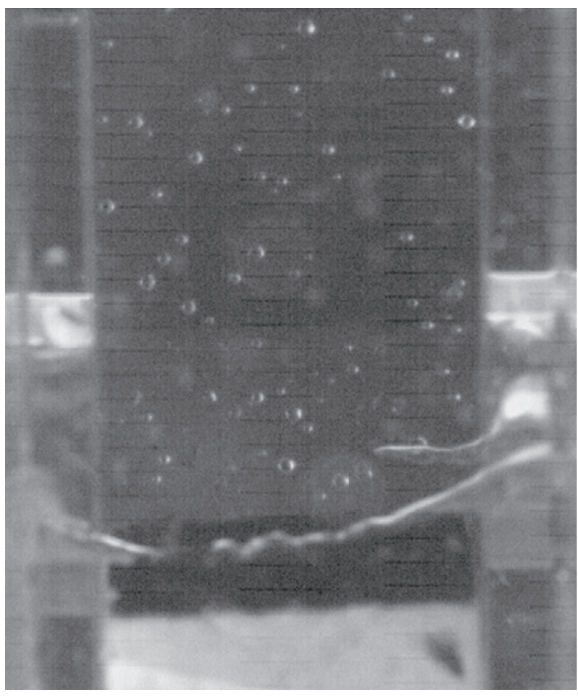

(c)

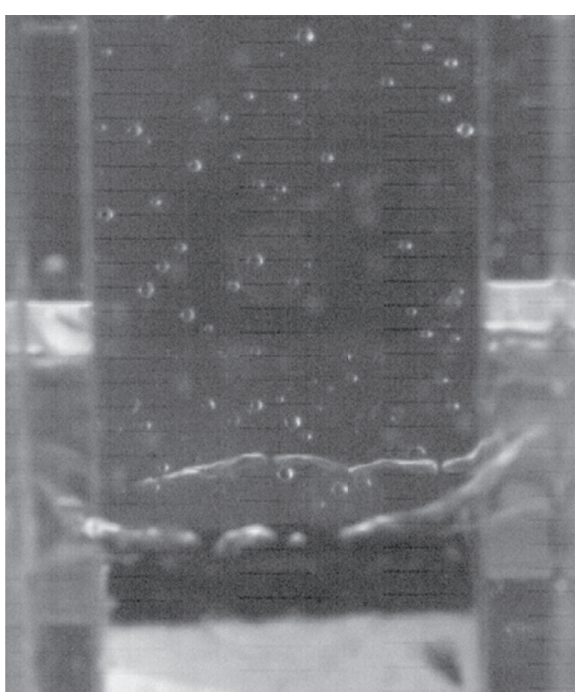

(b)

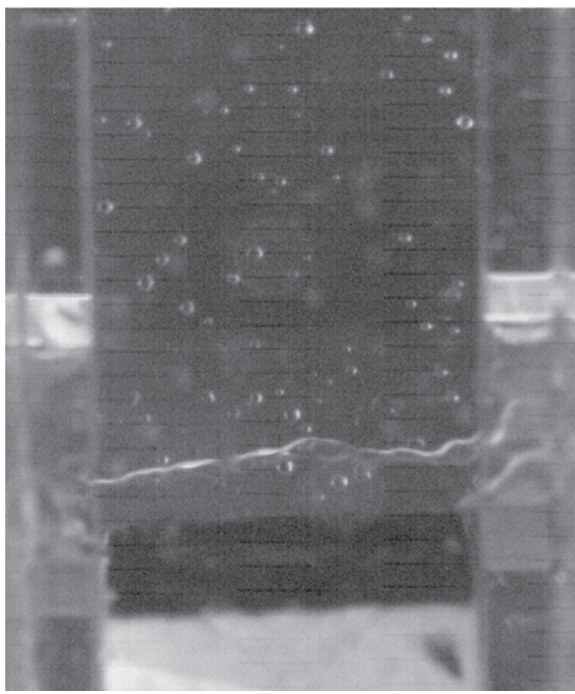

(d)

Figure 5: Dynamic evolution of the vortices inside the NIP, Case B, for a time interval of $0.036 \mathrm{~s}$.

characteristic is that there are instants where only one vortex is visible. Although the axes of rotation of both vortices are not straight lines, they are moderately parallel to each other and parallel to the nozzle exit ports' axis. These vortices arise because the pressure inside these zones decreases and thus become reverse flow zones that suction air through the exit ports. Figure 5 shows some representative examples of the shapes acquired by the two vortexes generated inside the nozzle without well. A plausible explanation of the vortex continuity breaking and the temporary absence of one vortex is that the pressure is higher than required inside the low-pressure zones.

High-speed video recordings registered air bubbles in the flow stream, one of them remained visible for $0.052 \mathrm{~s}$, and it was used as a tracer to estimate fluid velocity magnitude. Figure 6 depicts the trajectory of the bubble and the estimation of the fluid velocity as a function of the height relative to the bottom of the nozzle. The bubble travels near the NIP longitudinal axis with a constant velocity until it reaches the area near the exit ports. There, its speed decreases and the bubble moves away from the inner bore longitudinal axis. Later, the bubble accelerates when it passes in the middle of the two vortices and finally leaves the NIP at the bottom of the exit port.

The shapes, the sizes, and the dynamic behavior of the jets emerging from the nozzle without the pool are notably different in comparison with the nozzle with bottom well. Inspection of individual frames of video record suggests that despite the jets are not identical, the differences in sizes and shapes are quite small.

The jets emerging from the nozzle without a pool are compact in shape. Several authors [2, 21] named this 

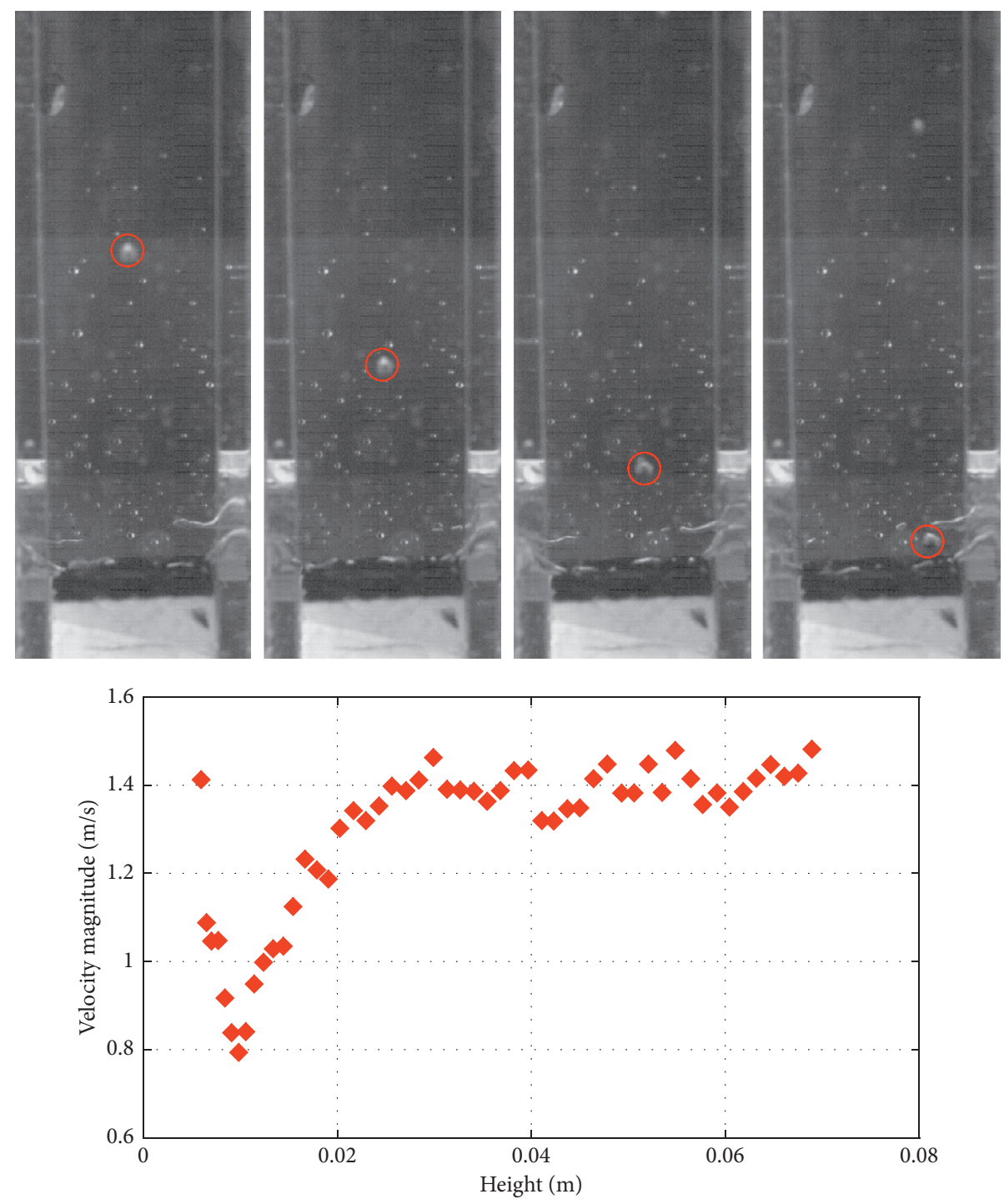

Figure 6: Bubble trajectory inside the NIP, Case B (top) and its measured velocity (bottom).

behavior as "smooth jets." The compact shape of the jets has two implications. The first one is related to the jet's impingement point. Due to their compact shape, the outlet jets do not collide directly with any of the mold walls and release much of their kinetic energy into the liquid steel within the mold. The second implication is that the compact shape of the outlet jets promotes uniform heat transfer between molten steel and mold walls. A practical implication of producing almost identical smooth outlet jets is a double-roll symmetric liquid steel flow pattern produced inside the mold. The distinctive characteristic of this flow pattern is the absence of extreme variations near the liquid steel-slag interface, which, in turn, decreases the undesirable slag trapping phenomenon.

2.4.2. Submerged Nozzle Condition. Figure 7 shows a visualization of the behavior of the fluid inside the NIP without pool using the "light-painting" technique. Compared with the previous NIP, Case A, visualization of the vortex is much more complicated because of the vortex intensity. This picture shows a close-up of the region near the exit port; a filter was applied to enhance visualization of particle trajectories. Two vortexes rest in the lower half of the volume defined between the two exit ports.

\section{Numerical Simulations}

3.1. Governing Equations and Turbulence Model Considerations. For developing the mathematical model, the following system properties were considered. The working fluid is incompressible and has Newtonian behavior. The studied process is isothermal, and all temperature-dependent physical properties remain constant. 


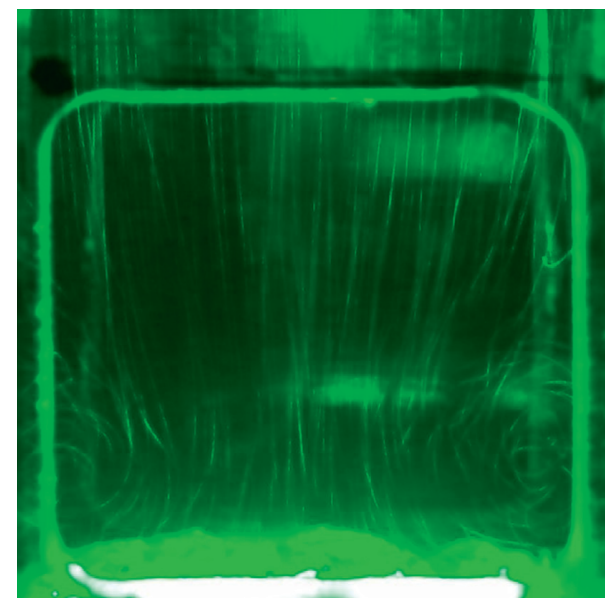

Figure 7: Two counterrotating vortexes located near the exit port of the NIP for the Case B.

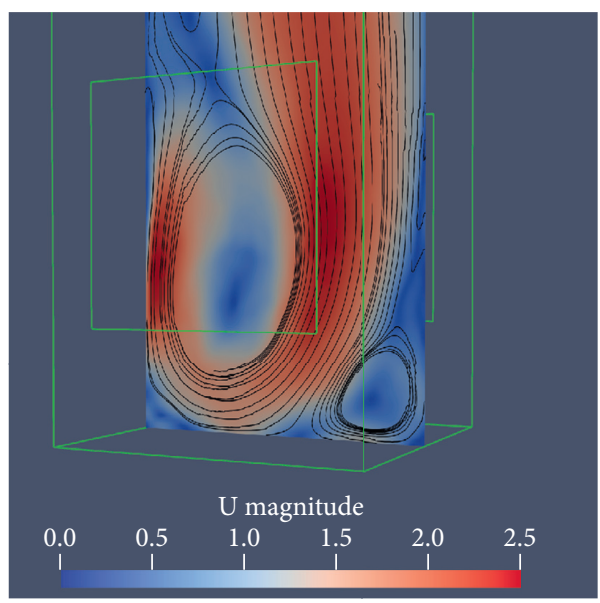

(a)

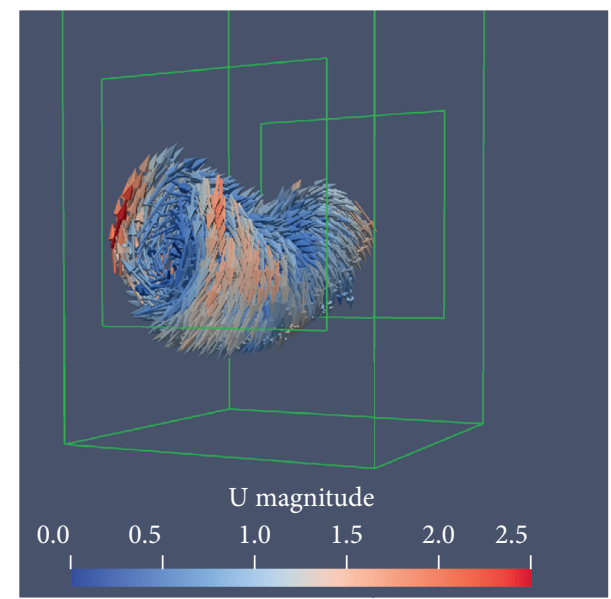

(b)

FIgUre 8: Turbulent fluid flow pattern inside the simulated NIP for Case A. (a) Plane parallel to nozzle exit ports colored by flow velocity magnitude and $2 \mathrm{D}$ streamlines and (b) vortex structure outlined by the fluid velocity vectors. The size of the vectors is the same, but they are colored according to their magnitude.

The governing equations of the fluid flow motion for incompressible fluid flow are the Navier-Stokes equations. However, for high Reynolds numbers, the adequate turbulence model is necessary to accurately reproduce the transient fluid flow behavior for a given system. Real et al. (2006) [12] compared the predictions of the fluid flow patterns inside a bifurcated SEN using two different turbulence models: the $(k-\varepsilon)$ and the large eddy simulation (LES) models. The authors found that the $(k-\varepsilon)$ model fails in recovering the transient behavior of the fluid flow inside the SEN observed experimentally.

Recently, Shukla and Dewan (2018) [13] presented a comparison of the prediction capabilities of four different subgrid stress (SGS) models for simulating the heat transfer in a slot jet impingement system with the Reynolds number of 20,000. The models analyzed in their work were Smagorinsky, WALE (wall-adapting local eddy-viscosity), $k$-equation, and dynamic $k$-equation. The authors of that work found that the velocity and turbulence profiles using the four LES models followed the trends of the experimental results. However, the authors also found that WALE and dynamic $k$-equation SGS models display superior performance in complex flow regions. Based on the preceding arguments, the transient numerical simulations presented in this work were done using an LES turbulence model with dynamic $k$-equation filtering.

The basic idea of LES is to directly resolve all turbulence scales larger than grid resolution in spatial and temporal while to model the effect of the turbulence scales smaller than that of the grid scale (GS) using the subgrid scale (SGS) model.

The LES turbulence model separates the fluid motion into small and large scales and directly resolves all turbulence scales larger than grid resolution while models the effect of the turbulence scales smaller than that of the grid scale using certain subgrid scale (SGS) model. The fluid 

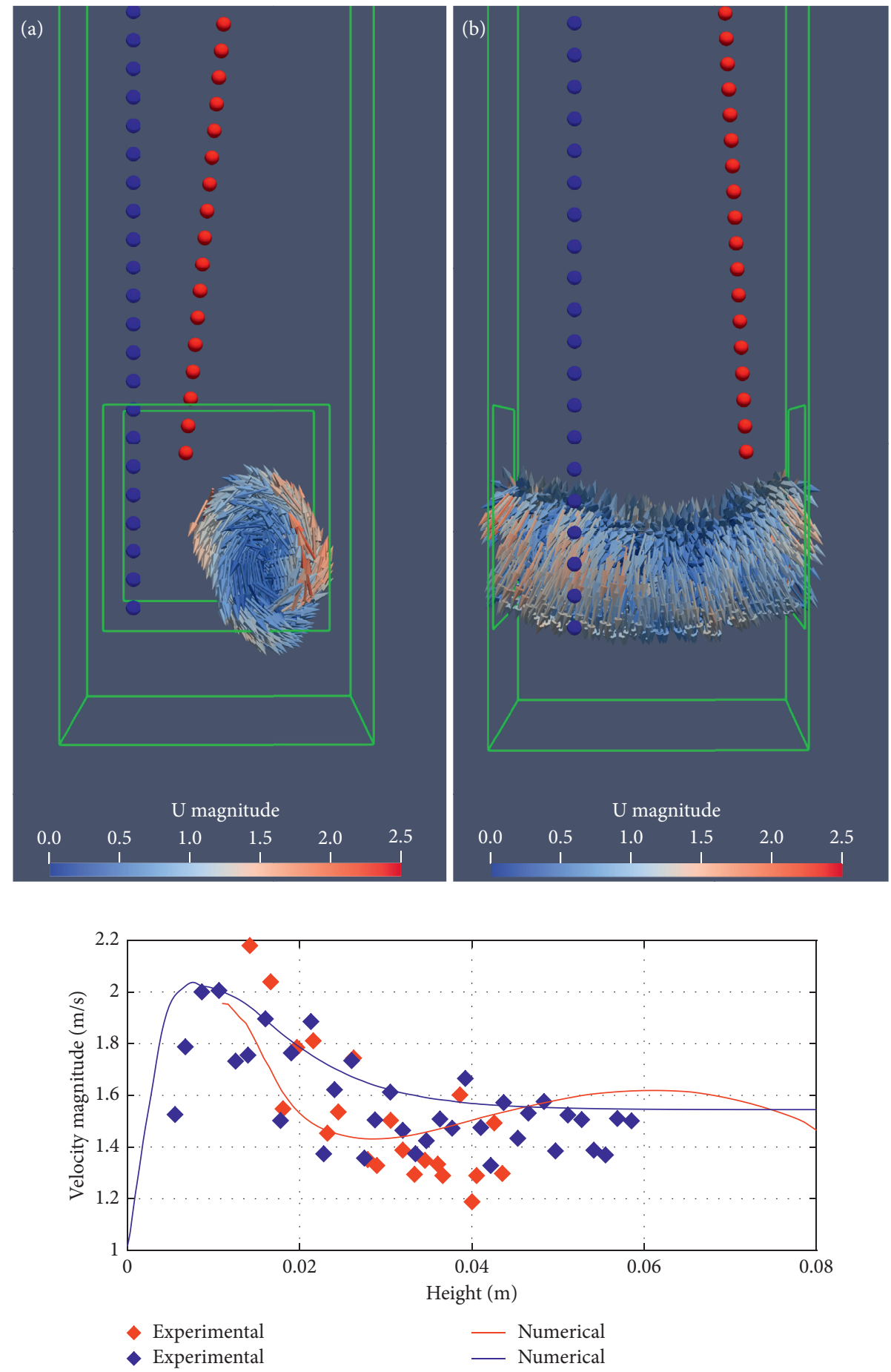

FIGURE 9: Vortex tube outlined by the velocity vectors shown in two projections (a) and (b) along with the paths used for velocity tracing. Bottom panel shows the comparison of experimental and numerical velocities.

motion separation is accomplished by means of a cutoff filter. The filtered governing equations are as follows:

$$
\begin{array}{r}
\frac{\partial \bar{u}_{i}}{\partial x_{i}}=0, \\
\frac{\partial \bar{u}_{i}}{\partial t}+\bar{u}_{j} \frac{\partial \bar{u}_{i}}{\partial x_{j}}=-\frac{\partial \bar{p}}{\partial x_{i}}-\frac{\partial T_{\mathrm{ij}}}{\partial x_{j}}+v \frac{\partial^{2} \bar{u}_{i}}{\partial x_{j} \partial x_{j}},
\end{array}
$$

where $\bar{u}_{i}$ is the filtered fluid velocity vector $i$-component, $\bar{p}$ is the filtered density-normalized pressure, and $v$ is the kinematic viscosity of the fluid. Equation (3) also includes the components of a SGS stress tensor $\mathbf{T}$ defined as follows:

$$
T_{\mathrm{ij}}=\overline{u_{i} u_{j}}-\bar{u}_{i} \bar{u}_{j}
$$

In the dynamic $k$-equation SGS model, the SGS stress tensor is modeled using the velocity-strain tensor, $\bar{S}$, the 


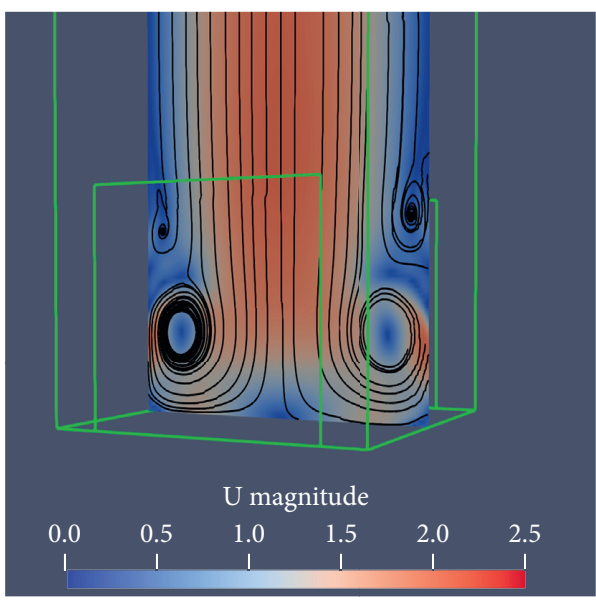

(a)

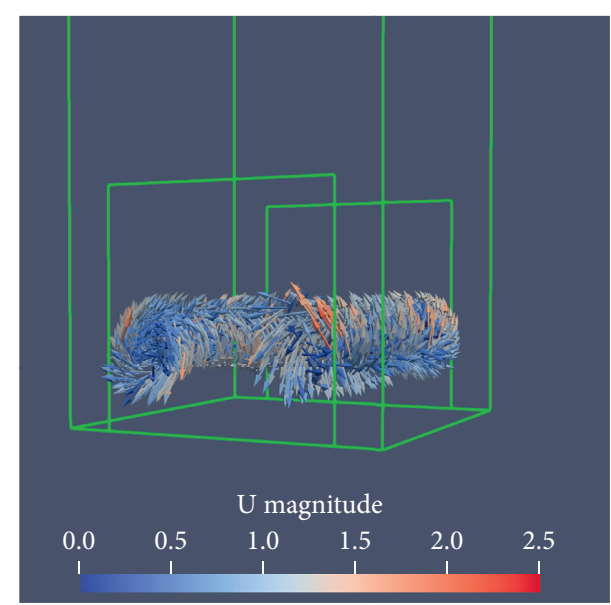

(b)

FIGURE 10: Turbulent fluid flow inside the simulated NIP for Case B. (a) Plane parallel to nozzle exit ports colored by flow velocity magnitude and 2D streamlines. (b) Two vortex structures outlined by the fluid velocity vectors. The size of the vectors is the same, but they are colored according to their magnitude.

subgrid scale kinematic eddy viscosity, $\nu_{\mathrm{SGS}}$, and the subgrid scale kinetic energy, $k_{\mathrm{SGS}}$ :

$$
\begin{aligned}
\bar{S} & =\frac{1}{2}\left(\frac{\partial \bar{u}_{i}}{\partial x_{j}}+\frac{\partial \bar{u}_{j}}{\partial x_{i}}\right), \\
T_{\mathrm{ij}} & =-2 \nu_{\mathrm{SGS}} \bar{S}_{\mathrm{ij}}+\frac{2}{3} k_{\mathrm{SGS}} \delta_{\mathrm{ij}}, \\
v_{\mathrm{SGS}} & =C_{k} \Delta \sqrt{k_{\mathrm{SGS}}} .
\end{aligned}
$$

In the previous equation, $\Delta$ is the subgrid characteristic length scale. In addition, $k_{\mathrm{SGS}}$ is obtained by solving the following transport equation:

$$
\frac{\partial k_{\mathrm{SGS}}}{\partial t}+\bar{u}_{i} \frac{\partial k_{\mathrm{SGS}}}{\partial x_{i}}=-T_{\mathrm{ij}} \frac{\partial \bar{u}_{i}}{\partial x_{j}}-C_{e} \frac{k_{\mathrm{SGS}}^{(3 / 2)}}{\Delta}+\frac{\partial}{\partial x_{i}}\left(v_{\mathrm{SGS}} \frac{\partial k_{\mathrm{SGS}}}{\partial x_{i}}\right) .
$$

This additional transport equation overcomes some drawbacks of algebraic eddy-viscosity models, which may occur in high Reynolds number flows and/or in the cases of a coarse grid model resolution [28]. This SGS model has two coefficients, $C_{k}$ and $C_{e}$, that must be calculated. Kim and Menon (1995) described in detail a localized dynamic formulation for obtaining these coefficients [29]. A remarkable feature of their formulation is its numerical consistency at high Reynolds numbers [30].

In this work, the OpenFOAM CFD toolbox was employed to accomplish numerical simulations. This computational program is an open-source software package and uses the finite volume method for numerical representation of the equations governing fluid motion. Several authors have recently shown that the program allows simulating a wide variety of complex fluid flow processes $[20,22-25,27]$. A detailed analysis of capabilities and validation study is presented in [17].
For solving the Navier-Stokes equations, OpenFOAM offers several methods to address the pressure-velocity coupling. This work used the PISO (pressure implicit with the split operator) method.

3.2. Numerical Simulation Setup. These are the boundary conditions for the mathematical model previously described:

(i) The inlet boundary condition was set as fully developed turbulent flow profile. The average inlet velocity is set to $\bar{u}_{z}=1 \mathrm{~m} / \mathrm{s}$.

(ii) The nonslip condition was considered for all the NIP solid walls $\left(u_{i}=0\right)$.

(iii) The inlet-outlet boundary condition was applied to both exit ports. Such condition switches to the inlet boundary if the local velocity vector next to the boundary is directed inside the domain (backward flow). This boundary condition allows to recover the reverse flow observed in physical experiments.

(iv) The nozzle discharges to the atmosphere.

In all the simulations, the convergence criterion is fulfilled when residuals for the modeled variables reached values of $1 \times 10^{-5}$. The convergence analysis was carried out using the results of the numerical simulations of the nozzle without the pool. The base for the convergence analysis was a model with a sufficiently large quantity of elements. Then, a second model was created by refining the mesh and increasing the amount of the elements. From the second model, a third model was built with four times the number of elements of the base model. We found that the hydrodynamic behavior obtained with the second numerical model entirely coincides with the results obtained in the physical simulations. The differences between the results of the numerical simulations with the second and the third model are negligible, so we take the mesh of the third model to carry out the numerical simulations. The third model also served as the base for creating the model for the nozzle with bottom 

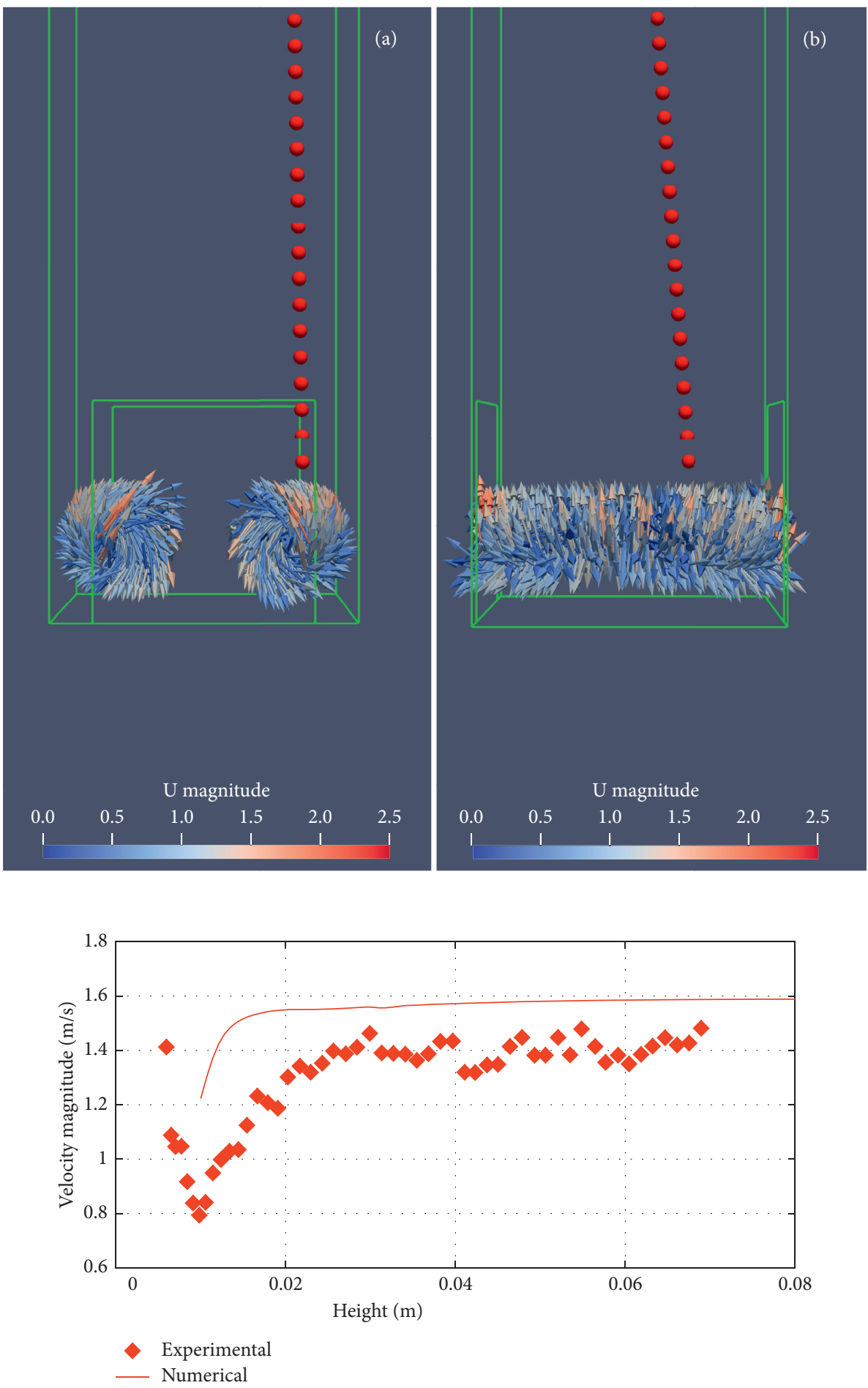

FIGURE 11: Vortex tubes outlined by the velocity vectors shown in two projections (a) and (b) along with the path used for velocity tracing. Bottom panel shows the comparison of experimental and numerical velocities.

well. In this case, the bottom well volume mesh has the elements of the same size as the elements in the central zone of the exit ports. The results of numerical simulations presented in this work are the average of 41 snapshots taken along an interval of two seconds.

3.3. Numerical Simulations: Case A. The results of numerical simulation of the NIP with the bottom well are shown in
Figure 8. The upper panel shows the velocity field and the streamlines along the plane perpendicular to the axis connecting exit ports, while the bottom panel shows the vortex surface where the fluid pressure is equal or lower than the atmospheric pressure along with the velocity vectors. This surface outlines the shape of the vortex produced inside the NIP. As can be noted from both panels, there is a dominant vortex that occupies a large fraction of the volume of the 
nozzle bottom. There is also a small vortical structure on the bottom of the well. Its rotation direction is opposite to the big vortex rotation direction. The rotation axis of the small vortex is almost parallel to the exit ports' axis. Over time, major changes were observed in the shape and size of the large vortex, but it was found that the vortex was always present.

Although the axis of the vortex is nearly parallel to the axis of the output ports of the NIP, it is never located on the nozzle central zone. The analysis of the numerical simulations confirmed that there is always a bias of the vortex axis with respect to the central plane of the NIP. A close inspection of the low-velocity vectors directed towards the interior of the NIP shows the existence of reverse flow. Note that the area near the center of the port has a very low velocity and liquid emerges with high velocity mainly along the edges of the exit ports. The latter fluid flow pattern suggests that the liquid emerges through disperse, coneshaped jets. This agrees with the findings obtained through physical simulations.

In order to investigate the velocity of the flow inside the NIP's bore, we extract the velocity magnitudes along two straight lines as depicted in the panels (a) and (b) of Figure 9. The bottom panel of Figure 9 shows the obtained velocities (blue and red lines) along with the measured velocities from the physical model (blue and red diamonds). It may be concluded that despite the different methods used for measuring velocities in both types of simulations, a qualitative agreement is obtained.

3.4. Numerical Simulations: Case B. Figure 10 shows an example of simulation where the two vortexes created inside the NIP without the pool. The analysis of the simulation led us to the following observations. The two vortexes located close to the bottom of the NIP are always present and preserve their cylindrical shape. Moreover, their axes remained roughly parallel to each other. The volumes of both vortexes oscillate continuously, tending, however, to preserve the similar size.

Figure 10 shows the magnitude of fluid velocity and the streamlines along the plane parallel to the exit ports (upper panel) and the zero-pressure surface of the vortexes together with the velocity vector magnitudes. The streamlines depict the presence of two dominant counterrotating vortexes located close to the bottom of the NIP and two smaller vortexes located just above the main ones. Overall, the velocity field is quite symmetric. As in the previous case, the low-velocity vectors indicate the presence of reverse flow inside the nozzle while high-velocity vectors are found close to the ports.

Notice that the velocity gradients are not as large as those observed in Case B. Furthermore, the largest velocity values are uniformly distributed at the bottom of the exit ports. This behavior agrees with smooth outlet jets found for the NIP without the pool.

Again, in order to compare the velocities inside the NIP obtained in the physical experiment with the numerical ones, we extract the velocity magnitude along a straight line as illustrated in Figure 11. As shown in the bottom panel, the agreement between both experiments is not as good as in the previous case. However, qualitatively, the velocity drop close to the bottom is reproduced, indicating the pattern of the smooth jets.

\section{Conclusions}

The nozzle internal prototype (NIP) studied in this work was constructed based on a box-type nozzle with an inner bore and a square cross section that remains constant throughout all its length. The NIP has two exit orifices of square geometric shape and satisfies the Froude similitude criterion.

In order to analyze hydrodynamics of the flow inside the nozzles, two different depths of pool inside the NIP were simulated using physical and numerical modeling. From the metallurgical industry perspective, Cases A and B reproduce two operational conditions. Case A shows the initial condition in the life cycle of the SEN, and Case B reproduces a condition when the liquid steel impurities were collected at the bottom zone of the nozzle.

The results show that the exit flow structure develops in the bottom zone of the nozzle; however, each case generates different turbulent flow conditions. Case A forms a single pronounced vortex that occupies a considerable area of the exit ports. Meanwhile, Case B develops dynamic flow behavior with two vortexes. The vortexes in both cases are located near the nozzle exit ports, and the reverse flow zones associated with them, emerge naturally, regardless of other factors.

The findings of this work are complementary to the information obtained using the circle cross section nozzle reported in 2006 [12] and are relevant for future works, especially for those regarding numerical simulations of inert gas injection in the stream flowing through the nozzle $[10,15,19]$.

An accurate evaluation of the gas injection effect first requires a deep analysis of the process performance without gas injection and the influence of other process components such as the sliding gate, for example. Another factor is the inlet flow misalignment; the effect of this process characteristic will become significant when the nozzle length is too short.

This study will allow to build nozzles that produce a symmetric, regular fluid flow pattern inside the mold, which leads to improvements on the process such as low energy consumption and finally in cost reductions.

\section{Data Availability}

The data used to support the findings of this study are available from the corresponding author upon request.

\section{Conflicts of Interest}

The authors declare that they have no conflicts of interest.

\section{Acknowledgments}

The authors would like to thank the Sistema Nacional de Investigadores-Conacyt for the distinction granted and the 
stipend received. Some of the physical simulations were developed in the Laboratorio de Ingenieria Termica e Hidraulica Aplicada (LABINTHAP), SEPI-ESIME, at the Instituto Politecnico Nacional. Numerical simulations and the rest of the physical simulations were developed in the Laboratorio de Computo y Visualizacion Cientifica, at Universidad Autonoma Metropolitana. Numerical simulations were done using OpenFOAM v6, the OpenFOAM Foundation. Visualizations of numerical simulations were done using ParaView v5.6.1. This work was partially supported by Projects of Universidad Autonoma Metropolitana number 22703022, PRODEP number 22711777, and Instituto Politecnico Nacional number SIP-20201315.

\section{References}

[1] G. E. Sellers, "Machinery for making pipes continuously from lead, \& C," US1908A, 1840.

[2] D. E. Hershey, B. G. Thomas, and F. M. Najjar, "Turbulent flow through bifurcated nozzles," International Journal for Numerical Methods in Fluids, vol. 17, no. 1, pp. 23-47, 1993.

[3] N. T. Mills and L. F. Barnhardt, "Development of submerged entry tundish nozzles," JOM, vol. 23, no. 11, pp. 37-43, 1971.

[4] I. Calderón-Ramos, R. D. Morales, R. Servín-Castañeda et al., "Modeling study of turbulent flow in a continuous casting slab mold comparing three ports SEN designs," ISIJ International, vol. 59, no. 1, pp. 76-85, 2019.

[5] H. Zhang, Q. Fang, T. Xiao, H. Ni, and C. Liu, "Optimization of the flow in a slab mold with argon blowing by divergent bifurcated SEN," ISIJ International, vol. 59, no. 1, pp. 86-92, 2019.

[6] D.-W. Li, Z.-J. Su, K. Marukawa, and J.-C. He, "Simulation on effect of divergent angle of submerged entry nozzle on flow and temperature fields in round billet mold in electromagnetic swirling continuous casting process," Journal of Iron and Steel Research International, vol. 21, no. 2, pp. 159-165, 2014.

[7] Z. Li, E. Wang, Y. Xu, and L. Xu, "Influence of SEN depth and port angle on vertical electromagnetic brake effects in continuous casting mould," COMPEL-the International Journal for Computation and Mathematics in Electrical and Electronic Engineering, vol. 36, no. 2, pp. 445-457, 2017.

[8] Z.-Q. Zhang, J.-B. Yu, Z.-M. Ren, and K. Deng, "Study on the liquid metal flow field in FC-mold of slab continuous casting," Advances in Manufacturing, vol. 3, no. 3, pp. 212-220, 2015.

[9] A. Asad, C. Kratzsch, and R. Schwarze, "Numerical investigation of the free surface in a model mold," Steel Research International, vol. 87, no. 2, pp. 181-190, 2015.

[10] Z. Wang, K. Mukai, and D. Izu, "Influence of wettability on the behavior of argon bubbles and fluid flow inside the nozzle and mold," ISIJ International, vol. 39, no. 2, pp. 154-163, 1999.

[11] D. Gupta and A. K. Lahiri, "Water modelling study of the jet characteristics in a continuous casting mould," Steel Research, vol. 63, no. 5, pp. 201-204, 1992.

[12] C. Real, R. Miranda, C. Vilchis, M. Barron, L. Hoyos, and J. Gonzalez, "Transient internal flow characterization of a bifurcated submerged entry nozzle," ISIJ International, vol. 46, no. 8, pp. 1183-1191, 2006.

[13] A. K. Shukla and A. Dewan, "OpenFOAM based LES of slot jet impingement heat transfer at low nozzle to plate spacing using four SGS models," Heat and Mass Transfer, vol. 55, no. 3, pp. 911-931, 2018.

[14] C. A. Real-Ramirez and J. I. Gonzalez-Trejo, "Analysis of three-dimensional vortexes below the free surface in a continuous casting mold," International Journal of Minerals, Metallurgy, and Materials, vol. 18, no. 4, pp. 397-407, 2011.

[15] Z.-Q. Liu, F.-S. Qi, B.-K. Li, and M.-F. Jiang, "Vortex flow pattern in a slab continuous casting mold with argon gas injection," Journal of Iron and Steel Research International, vol. 21, no. 12, pp. 1081-1089, 2014.

[16] K. K. Chen, C. W. Rowley, and H. A. Stone, "Vortex dynamics in a pipe T-junction: recirculation and sensitivity," Physics of Fluids, vol. 27, no. 3, 2015.

[17] E. Robertson, V. Choudhury, S. Bhushan, and D. K. Walters, "Validation of OpenFOAM numerical methods and turbulence models for incompressible bluff body flows," Computers \& Fluids, vol. 123, pp. 122-145, 2015.

[18] J. Gonzalez-Trejo, C. A. Real-Ramirez, R. Miranda-Tello, F. Rivera-Perez, and F. Cervantes-De-La-Torre, "Numerical and physical parametric analysis of a SEN with flow conditioners in slab continuous casting mold," Archives of Metallurgy and Materials, vol. 62, no. 2, pp. 927-946, 2017.

[19] S. Sarkar, V. Singh, S. K. Ajmani, R. K. Singh, and E. Z. Chacko, "Effect of argon injection in meniscus flow and turbulence intensity distribution in continuous slab casting mold under the influence of double ruler magnetic field," ISIJ International, vol. 58, no. 1, pp. 68-77, 2018.

[20] G. R. Tabor and M. H. Baba-Ahmadi, "Inlet conditions for large eddy simulation: a review," Computers \& Fluids, vol. 39, no. 4, pp. 553-567, 2010.

[21] S. Pirker, D. Kahrimanovic, and S. Schneiderbauer, "Secondary vortex formation in bifurcated submerged entry nozzles: numerical simulation of gas bubble entrapment," Metallurgical and Materials Transactions B, vol. 46, no. 2, pp. 953-960, 2015.

[22] Y. Cao and T. Tamura, "Large-eddy simulations of flow past a square cylinder using structured and unstructured grids," Computers \& Fluids, vol. 137, pp. 36-54, 2016.

[23] E. Fooladgar, C. K. Chan, and K.-J. Nogenmyr, "An accelerated computation of combustion with finite-rate chemistry using LES and an open source library for in-situadaptive tabulation," Computers \& Fluids, vol. 146, pp. 42-50, 2017.

[24] J. Mao, K. Zhang, and K. Liu, "Comparative study of different subgrid-scale models for large eddy simulations of magnetohydrodynamic turbulent duct flow in OpenFOAM," Computers \& Fluids, vol. 152, pp. 195-203, 2017.

[25] J. Mao, Y. Yin, L. Yu, K. Xiang, and H. Wang, "Large eddy simulation of geometry sensitivity of magneotohydrodynamic turbulent flow in a rectangular duct," Fusion Engineering and Design, vol. 127, pp. 111-119, 2018.

[26] C. A. Real-Ramirez, I. Carvajal-Mariscal, F. Sanchez-Silva, F. Cervantes-De-La-Torre, J. Diaz-Montes, and J. GonzalezTrejo, "Three-dimensional flow behavior inside the submerged entry nozzle," Metallurgical and Materials Transactions B, vol. 49, no. 4, pp. 1644-1657, 2018.

[27] M. Kim, J. Lim, S. Kim, S. Jee, J. Park, and D. Park, "Largeeddy simulation with parabolized stability equations for turbulent transition using OpenFOAM," Computers \& Fluids, vol. 189, pp. 108-117, 2019.

[28] S. Huang and Q. S. Li, "A new dynamic one-equation subgridscale model for large eddy simulations," International Journal for Numerical Methods in Engineering, vol. 81, no. 7, pp. 835-865, 2010.

[29] W.-W. Kim and S. Menon, "A new dynamic one-equation subgrid-scale model for large eddy simulations," in Proceedings of the 33rd Aerospace Sciences Meeting and Exhibit, 
American Institute of Aeronautics and Astronautics, Reno, NV, USA, January 1995.

[30] S. Menon, P.-K. Yeung, and W.-W. Kim, "Effect of subgrid models on the computed interscale energy transfer in isotropic turbulence," Computers \& Fluids, vol. 25, no. 2, pp. 165-180, 1996.

[31] K. K. Chen, C. W. Rowley, and H. A. Stone, "Vortex breakdown, linear global instability and sensitivity of pipe bifurcation flows," Journal of Fluid Mechanics, vol. 815, pp. 257-294, 2017.

[32] M. Kahshan, D. Lu, and M. Rahimi-Gorji, "Hydrodynamical study of flow in a permeable channel: application to flat plate dialyzer," International Journal of Hydrogen Energy, vol. 44, no. 31, pp. 17041-17047, 2019.

[33] D. Vigolo, S. Radl, and H. A. Stone, "Unexpected trapping of particles at a $T$ junction," Proceedings of the National Academy of Sciences, vol. 111, no. 13, pp. 4770-4775, 2014. 